Pensamiento Crítico No. 8, pag. 1 - 12

\title{
Una Aproximación, a los Mecanismos para la Determinación de los Precios de los Metales \\ Ysaac Ospino Edery
}

\begin{abstract}
Resumen: Es sabida la relevancia que tiene la actividad minera para la economía de nuestro país, la misma que en estos dos últimos años como consecuencia de la subida de los precios de los metales registra un incremento sustantivo de su valor, representando alrededor del $60 \%$ del total exportado. Si bien este porcentaje nos puede resultar gratificante, también nos indica ya cuan vulnerable y dependiente es nuestra economía primario exportadora frente a los altibajos cíclicos del mercado mundial.
\end{abstract}

Este trabajo se limita a tocar aspectos referidos a los mecanismos para la determinación de los precios de los metales en las Bolsas (LME y COMEX) y otras organizaciones especializadas (Metals Week, Metal Bulletin) que son tomadas como referentes en los contratos de compraventa para la comercialización minera metalúrgica que es la razón del negocio de la inversión minera.

Las cotizaciones que se constituyen en el LME y el COMEX así como en las revistas especializadas MW y MB son utilizadas por la gran mayoría de países, sin que se realicen estas transacciones directamente en las Bolsas de Metales. Siendo evidente que estos precios dependan de ciertos factores, como el de la demanda del metal que esta en función de la mayor o menor expansión económica de los países consumidores, teniendo en cuenta los factores políticos y sociales que los acontecen a nivel mundial. Es en estas situaciones que las Bolsas de Metales cobran su importancia como organismos reguladores del precio de los metales. 


\section{Ysaac Ospino Edery}

Palabras claves: Precio del metal, bolsas de metales, formula para valorización de concentrados, publicaciones especializadas.

\section{Las Cotizaciones de los Metales en el Mercado Mundial}

Frecuentemente tomamos conocimiento que los precios de los metales subieron o bajaron por diferentes factores, los cuales no dependen del comportamiento económico de nuestro país sino que los mismos están sujetos entre otros a determinados mecanismos que es importante el conocer como operan, dado que en las diferentes operaciones de compraventa interna y externa que realiza la pequeña, mediana o gran minería de nuestro país ya sea de minerales concentrados o refinados. Se utilizan fórmulas para la valoración de los mismos, teniendo como referencia a las cotizaciones que se determinan en las Bolsas de Metales u otras organizaciones que cumplen una función similar. (Ver tablas A, B, C).

"Los concentrados cotizan a los precios de los metales menos descuentos por costos de tratamiento, pérdidas y otros factores. Las siguientes fórmulas permiten determinar el precio que se paga por los concentrados".

TABLA: A

$\begin{array}{ll}\text { Fómula } A: & V C=[M-D]\left[P . F-\left(R-+E_{1}\right)\right]-\left(T-+E_{2}\right)-X+Y \\ \text { Fómula } B: & V C=[M-D][P . F]-[(T-+E)+T(P-P B)]-X+Y\end{array}$

Donde:

VC: Valor del Concentrado.

M : Contenido de Metal.

D : Deducciones por perdida metalúrgica.

P : Precio del Metal (cotización referencial a utilizar).

F : Factor del Precio.

$\mathrm{R}$ : Maquila de Refinación (Refining Charge).

$\mathrm{E}_{1}$ : Escalador de Maquila de Refinación.

$\mathrm{T}$ : Maquila de Tratamiento.

$\mathrm{E}_{2}$ : Escalador de Maquila de Tratamiento.

PB: Precio Base referente al Costo de Tratamiento (Fundición y/o Refinación).

$\mathrm{T}$ : Factor de Ajuste del Costo de Tratamiento.

$X$ : Deducciones por Impurezas.

$Y$ : Créditos por Subproductos. 
Una aproximación, a los mecanismos para la determinación de los precios de los metales

TABLA: B

Contrato de compraventa de Concentrados de Cobre entre DOE RUN PERU SRL y CENTROMIN PERÚ, AÑO 2003, solo se ha tomado por motivo didáctico la cláusula del precio (pagos).

\section{"Precio"}

Cobre: $100 \%$ del contenido final de cobre en el producto, previa deducción 1.1 unidades, al promedio de la cotización "LME Grade A" Settlement para el cobre, como se publica en la Revista Metals WeeK, para el periodo de cotización aplicable.

Plata: $95 \%$ del contenido final de plata en el producto, sujeto a una deducción mínima de 30 gramos por tonelada métrica seca, al promedio de la cotización London Spot, como se publica en la revista Metals Week, para el periodo de cotización aplicable.

Oro: $95 \%$ del contenido fino de oro en el producto, sujeto a una deducción mínima de 1 gramo por tonelada métrica seca, al promedio de las cotizaciones London Initial y London Final para el oro, como se publican en la revista Metals Week para el periodo de cotizaciones aplicable.

TABLA: C

"Contrato de Venta de Telurio Refinado (siendo un contrato valido no se considera los nombres de el comprador y vendedor)".

Contrato Nro. 1000

This International Sales Agreemmet entered in to by and between xxxxSRL as seller, with domicile at $x x x x$, and $x x x x$ CO.Ltd, as Buyer, with domicile at $x x x x$, China in the following terms and conditions:

1. Product: Tellurium Metal Stiks CP brand Peruvian origin $99.80 \%$ minimum purity.

2. Quantity: $500 \mathrm{Kgs}+/-2 \%$ approximately.

3. Delivery: CIF Hong Kong - China (In one conteiner- FCL-FCL). Aaccording to Incoterms Last Edition 2000.

4. Shipments: From Callao - Peru, end Febrery 2004, subjet to maritime space availibility.

5. Price: US $\$ 85.00 / \mathrm{kg}$ (Reference Metall Bulletin), CIF Hong Kong.

6. Quotational Period: Fixed Price.

7. Payment: $100 \%$ in US Dollars, Cash Agaist Document (CAD).

8. Others Conditions (Ceteris Paribus). 


\section{Algunas condiciones del Mercado}

Los precios de los minerales concentrados y refinados más representativos para nuestra economía, (cobre, plomo, plata, zinc, oro / llámense metales) son comúnmente el reflejo de las condiciones que imperan en cada momento en el mercado mundial. En el cual participan compradores y vendedores de toda magnitud, influenciando de manera decisiva en las fluctuaciones y la estabilidad de los precios.

Es así como los grandes compradores de EE.UU, Japón, RFA, China, y otros (cuadro $\mathrm{N}^{\circ}$ 1), juegan un papel decisivo en el funcionamiento del mercado mundial donde los efectos de las situaciones políticas, económicas, financieras que atraviesan estos países, repercuten en los precios de los metales y en las expectativas futuras en cuanto a las cotizaciones.

\section{CUADRO No 1}

Consumidores de Refinados (Miles TM)

\begin{tabular}{|l|c|c|c|c|}
\hline \multicolumn{1}{|c|}{ PAIS } & COBRE & ZINC & PLOMO & ESTAÑO \\
\hline EE.UU. & 2,594 & 1,107 & 1694 & 50 \\
CHINA & 2,207 & 1512 & 643 & 62 \\
JAPON & 1,146 & 633 & 321 & 22 \\
ALEMANIA & 1,092 & 531 & 401 & 23 \\
COREA DEL SUR & 849 & 394 & 312 & 14 \\
ITALIA & 676 & 355 & 283 & ND \\
TAIWAN & 540 & 276 & ND & ND \\
FRANCIA & 538 & 336 & 254 & ND \\
MEXICO & 445 & ND & 243 & ND \\
BRASIL & 345 & ND & ND & ND \\
INDIA & ND & 278 & ND & ND \\
\hline
\end{tabular}

Fuente: Estimado al 2003 US GEOLO GICAL SURVEY, Elaboración propia.

El mercado de los metales (refinados) tiene un funcionamiento complejo, tal es así que en la fijación de su precio es indudable que se tenga en cuenta tanto el volumen ofertado por parte de los productores, y la demanda por parte de os consumidores (industriales), que encuentran en el mercado un precio que les permite perfeccionar un negocio de compra y venta; pero no siempre los precios que rigen el mercado reflejan la situación tanto de la oferta y demanda. Cabe resaltar que el mercado de concentrados para nuestro país resulta el de mayor envergadura dado 
Una aproximación, a los mecanismos para la determinación de los precios de los metales

que de esta calidad son los mayores volúmenes que exportamos a fundiciones y refinerías que son en número mucho menor que los compradores de refinados.

"En muchos casos las cotizaciones se modifican como consecuencia de muchos factores y medidas unilaterales que en la práctica se allegado a realzar por parte de los productores (vendedores). Operaciones de compra en las Bolsas de Metales, con la finalidad de mantener el mercado y sostener un precio más o menos estable cuando la demanda ha sido débil o viceversa" 1 .

Es notorio que los factores que afectan la fijación de los precios de los metales, puedan darse por un exceso de producción del metal, el cual se hace sentir rápidamente en el mercado, igualmente un aumento violento en la demanda influye de inmediato en los precios que se registran.

A los factores mencionados, se suman otros que en gran parte influencian directamente en la generación de los precios al contado y a futuro, como es la existencia de metales en stock, en poder de los productores, comerciantes, consumidores y en las Bolsas de Metales.

De manera adicional la calidad del comprador o vendedor, concediendo descuentos, premios de diversa índole, facilidades de pago, así como los aspectos ambientales y otros.

\section{Las Bolsas de Metales y las Publicaciones Especializadas}

En el ámbito internacional se conocen centros importantes, destacándose el London Metal Exchange (LME) y el Comodity Exchange Inc (COMEX), ubicados en la ciudad de Londres y New York respectivamente. Existen además otros mercados determinados, que reflejan lascondiciones particulares de cada uno de ellos, que en cierta medida establecen la conformación de un precio que puede ser el promedio ponderado del valor de los diferentes suministros que contribuyen a su abastecimiento.

Así tenemos el precio Girm en Francia, el precio interno Delnotiz en Alemania, el precio de fundidores de Japón, el precio de comerciantes en EE.UU, el precio de productores del Canadá, el precio de productores de Bruselas, etc.

También existen algunas publicaciones especializadas que periódicamente dan a conocer 


\section{Ysaac Ospino Edery}

ciertos promedios de precios referidos a una base FOB o CIF, y que representan el valor ponderado de operaciones efectivas (ventas realizadas). Haciendo la distinción según provengan de producciones internas o foráneas, con relación a estas, existen en New York el semanario "Metals Week" que publica los precios "Domestic Refinery" y "Export Refinery", a la que podemos tener acceso previo pago por suscripción.

En Londres se publica la revista "Metal Bulletin" que hace algo similar al precio "Export Refinery" de la revista norteamericana pero sobre una base CIF Londres. Frecuentemente son usados en contratos de compraventa y los datos que consignan las mismas, son tomados directamente de las Bolsas de Metales o de las fuentes primarias. En cuanto a la producción de metales que nos compete, son tomados como referentes para la venta de los Metales Menores "Minors Metals", Bismuto, Selenio, Telurio, Indio, Cadmio, etc.

Las transacciones que se realizan a través de las Bolsas de Metales, presuponen la absoluta libertad de traslado de las mercaderías dentro y fuera de los límites en que se encuentran las bodegas oficiales (Gran Bretaña, Holanda, Alemania, Bélgica, etc.). De igual libertad debe disponerse para hacer efectivos los créditos $u$ órdenes de pago a través de los bancos. Para los deterioros o pérdidas debe contarse con las necesarias empresas aseguradoras.

En las Bolsas de Metales se dan transacciones efectivas que se van a caracterizar porque se hace entrega física del metal. Y aquellas que son meramente bursátiles en las que las mercaderías desempeñan un papel secundario y el interés radica en realizar dispares operaciones cuando existe diferencia de precios.

"Las transacciones efectivas pueden ser al contado (cash) cuando el material (metal) debe estar disponible para su entrega inmediata (día siguiente), desde las bodegas oficiales reconocidas por las Bolsas. A plazo (transacción futura), en la que existe cierto plazo para elegir la entrega física del metal. La ventaja principal de este tipo de negocio radica en que permite al comprador (industrial elaborador) conocer con anticipación el costo de su materia prima y disponer su plan de producción y financiamiento de acuerdo con este antecedente. Al mismo tiempo durante el plazo hay un período para que los productores de metales puedan trasladarlo desde los establecimientos mineros, a las bodegas autorizadas por la Bolsa"².

En general, las transacciones que se practican en las Bolsas de Metales van a cumplir una 
Una aproximación, a los mecanismos para la determinación de los precios de los metales

función de regulación del precio del metal a nivel Mundial. En base al cual los agentes compradores y vendedores se ponen de acuerdo para realizar sus operaciones comerciales. Los contratos con los cuales se oficializa las transacción son similares entre sí (formato estándar) y en ellos se hace constar los arreglos necesarios en torno al negocio acordado, para tener que evitar dilucidar disputas recurriendo a la justicia ordinaria.

\section{Operaciones de las Bolsas de Metales}

Las principales acciones son las siguientes:

A. Operaciones de compra y venta al contado (cash), lo que equivale a que un productor poseedor venda el metal a un consumidor (interesado), el metal físico debe estar disponible en una de las bodegas reconocidas por la Bolsa.

B. Contratos de compraventa a plazos (futuro), para entrega a futuro, sujetos a los términos y condiciones especificadas por la misma.

C. Operaciones de arbitraje, vienen a ser la compraventa simultanea de cantidad equivalente del producto en dos mercados diferentes, generalmente Londres y New York. Esto se hace cuando la estructura de precios de un mercado está fuera de línea con su contraparte en el exterior. Deduciéndose la diferencia, que viene a constituir los premios pagados por productos de calidad superior a la calidad del contrato, y los descuentos cobrados por calidades inferiores, estos diferenciales se indican en el contrato, para cubrir los fletes, etc.

D. Operaciones especulativas, tienen por objeto obtener utilidades en la Bolsa, basadas en los pronósticos del mercado y su consecuente variación de precios.

E. Operaciones de postergación, tienen su aplicación cuando antes del plazo fijado para el vencimiento de una operación de cobertura, el vendedor no desea entregar el metal físico, o el comprador no desea recibirlo. Ambos de común acuerdo prolongan el plazo perfeccionando su posición mediante un contrato.

\section{The London Metal Exchange (Bolsa de Metales de Londres) LME}




\section{Ysaac Ospino Edery}

En el ámbito mundial las transacciones de compra y venta de metal a través de un organismo común se realizaron desde 1869, la "Lombard Exchange and Newsroom".

"La intensificación del comercio de los metales en los años subsiguientes, permitió que tanto la "London Exchange Company" y la "London Metal Market", se fusionaron en 1881 formando el (The London Metal Market and Exchange Co. Ltda.), sobre cuyas bases se dio origen a la Bolsa de Metales de Londres (LME) en 1862" ${ }^{3}$.

"Las transacciones que se efectuaban en lo primero años de su funcionamiento se centraban principalmente es estaño y cobre, tomándose como unidad de medida para los contratos de compraventa de cobre las barras chilenas (Chile Bars) por ser Chile en esa época el único exportador de cobre" $"$.

La Bolsa de Metales de Londres, a medida que se intensificaba las transacciones comerciales de metales, fue convirtiéndose en el centro mundial del comercio de los mismos a partir de 1919, os sea después de al a Primera Guerra Mundial, durante la cual la Bolsa dejó de operar (1914-1918).

Es a partir de 1919 en que la Bolsa se constituyó en el eje central de la comercialización de metales (a escala mundial) dejando de funcionar durante la II Guerra Mundial, siendo a partir de 1953 en que su funcionamiento se ha realizado en forma continua hasta la fecha, convirtiéndose en el principal referente para la toma de las cotizaciones que allí se registran diariamente.

Desde enero del año 2000, The London Metal Exchange Limited se convirtió en una empresa subsidiaria, propiedad de LME Holding Limited.

Las Ruedas en la Bolsa de Londres, como en todas las bolsas de comercio para poder cerrar las operaciones de compraventa, se realizan diariamente en reuniones denominadas Ring (Ruedas). Estas se efectúan en LME en un círculo (diámetro de $10 \mathrm{mts}$ ) donde los corredores oficiales se ubican y efectúan transacciones de las partidas de metales a viva voz, las cuales posteriormente son perfeccionadas mediante un contrato especial escrito en un formulario standard.

El LME, tiene como característica principal de que es un mercado exclusivo para sus 
Una aproximación, a los mecanismos para la determinación de los precios de los metales

miembros, es decir que todo aquel que quiera participar en sus sesiones, deberá realizar operaciones en última instancia a través de un miembro de la 1ra categoría (Ring Dealing Member) "El MLE tiene 5 tipos de miembros, pero sólo los que pertenecen al grupo $\mathrm{N}^{\circ} 1$, tienen derecho a sentarse alrededor del Ring".

Las sesiones se realizan de lunes a viernes desde las 9 de la mañana hasta las 6:30 de la tarde en el local principal de la Bolsa, llevándose a cabo operaciones comerciales de cobre, zinc, plata, plomo, estaño, etc en horarios determinados para cada metal.

Los requisitos para efectuar las transacciones comerciales son numerosos, los principales vienen a ser:

- Se realizan transacciones con un mínimo de tonelajes, las cantidades de mayor envergadura tienen que ser múltiples de la cantidad mínima (para el caso del cobre, plomo, zinc, aluminio, la mínima es de $25 \mathrm{tn}$ ).

- Solamente son comerciales (al interior de la Bolsa), aquellos metales cuya marca de fabricación se encuentre registrada y aceptada en la Bolsa.

- Los volúmenes del metal tienen que garantizar la condición anterior de manera permanente, para el caso del cobre este tiene que ser del tipo Wire Bars Electrolítico, de dimensiones standard y un peso de 110 a $125 \mathrm{Kg}$. con una pureza de $99.99 \%$ de cu.

\section{La Formulación del Precio}

"Al finalizar las sesiones de la mañana en la rueda (1:00 PM), se anuncian los precios oficiales que regirán para el día, su oficialización se registra por intermedio del Secretario de la Bolsa, y la determinación del precio del metal es realzada por "Comité" de precios, el cual está integrado por tres miembros de la rueda" ${ }^{5}$.

Para la determinación del precio del metal se tiene en cuenta:

- Las cotizaciones de las diversas transacciones que se efectúan durante la reunión de la mañana (ring/rueda). 


\section{Ysaac Ospino Edery}

- La facultad de desconocer alguna oferta que se considere contraria a las normas establecidas y su dictamen final, viene a constituir los precios oficiales del metal que regirán a nivel mundial.

\section{Las Bodegas Oficiales}

Para el cumplimiento de los contratos de compraventa, en cuanto se refiere a la entrega física, el LME cuenta con bodegas y depósitos oficiales, los que pertenecen a empresas particulares debidamente autorizadas por el comité ejecutivo del LME y sujetas a disposiciones que rigen la Bolsa, evitando que mediante la especulación se llegue a distorsionar el funcionamiento de ésta, localizándose en Inglaterra, algunos países de Europa y al este de Asia. Las entregas de las bodegas oficiales de almacenamiento de los metales se hacen mediante certificados de depósito (warrant), el cual puede ser negociado con terceras personas.

\section{Bolsa de Metales de Nueva York (COMEX/NYMEX)}

Esta Bolsa que funciona en el mercado de los EE.UU, guarda similitud con la forma organizativa, funcionamiento y fines del LME.

Las transacciones de compraventa de metales se realizaban en forma conjunta con otras materias primas dentro de las operaciones comerciales y generales de la "New York Stock Exchange" a mediados del siglo XIX. Es a partir de la creación de la "Comodity Exchange Inc" (COMEX), que las operaciones comerciales de metales se constituye en su actividad principal ente otras. La Segunda Guerra Mundial truncó por algunos años su funcionamiento por la intervención del gobierno sobre las existencias y los precios a través de un control estricto.

A pesar que el COMEX es una entidad de carácter particular está sometida al control de la Oficina Fiscal General de los E.U. (Segurities and Exchange Commissions).

Hoy en día el COMEX es una división de la Bolsa Mercantil de New York (New York Mercantile Exchange / NYMEX), considerado como uno de los mayores mercados en el ámbito mundial de futuros y opciones sobre metales, con contratos de cobre, aluminio, oro y plata entre otros. 
Una aproximación, a los mecanismos para la determinación de los precios de los metales

\section{A Manera de Conclusión}

El LME y el COMEX, así como las publicaciones especializadas Metals Week (MW) y Metal Bulletin (MB), se constituyen en organismos, donde la formulación de los precios de los metales se da por acción de operaciones de compraventa por un volumen total anual pequeño, comparado con el metal comercializado en el resto del mundo. Pero sus cotizaciones son utilizadas por la gran mayoría de países, sin que se realicen estas transacciones directamente en las Bolsas de Metales o en las plazas donde se las toma.

Siendo evidente que estos precios dependan de ciertos factores, como el de la demanda del metal que esta en función de la mayor o menor expansión económica de los países consumidores, teniendo en cuenta los factores políticos y sociales que los acontecen. Es en estas situaciones que estas organizaciones cobran su importancia como organismos reguladores del precio de los metales.

Es indudable que no es un mecanismo completamente acabado, cuenta con algunos vacíos en su estructura y funcionamiento por ser pequeños los volúmenes que así se comercializan. Pero en la actualidad su vigencia es necesaria en la determinación de las cotizaciones de los metales, ya que permite la realización de transacciones comerciales a nivel mundial como sujeto referente en al concretización de los contratos de compraventa.

\section{Notas Referenciales}

1 Administración de Empresas Mineras y Comercio de Minerales y Metales, Robert Muller Hess, II parte pag. 164-169.

${ }^{2}$ IBID anterior

${ }^{3}$ IBID anterior

4 Wolf Frederick F. Conferen sobre el London Metal Exchange (Presidente de LME), Agosto 1973.

${ }^{5}$ IBID anterior 


\section{Ysaac Ospino Edery}

\section{BIBLIOGRAFÍA}

Juan Cristóbal Ciudad: "Deteminantes del precio spot del cobre en las Bolsas de Metales”. CEPAL-Cochilo. División de Recursos Naturales, Santiago de Chile 2005.

Alfredo Dammert Lira: "Economía Minera”. Minero Perú Comercial 1990.

Bureau of Mines: US Departament Of Interior "Mineral Facts And Problems", Washington DC. 2003.

Metalgesslschaft: "Metal Statistics" Frankfurt 2004.

The London Metal Exchange Limited: Plantation House / Fenchurch Street London, Fax 071-626-1703.

Juan José Ospino Edery: "La Incidencia del Mercado Mundial en La Producción Nacional de Cobre", Tesis, Facultad de Economía UNMSM.

Memoria Anual BCR: "Banco Central de Reserva del Perú” 2000-2006.

Robert Muller Hess: "Administración de Empresas y Comercialización de Metales y Minerales”, Editora Hispanoamérica, Chile 1970-2000.

Strauus Simón: "Comercialización de Metales" Editora. Office. San Francisco, EE.UU. 1974-2002.

Guillermo Diaz Gazani: "Que esperar de los precios en el Mercado Internacional", Estudios Económicos, BWS, 2004.

The Silver Institute: "World Silver Survey 2007 A Summary".

Ministerio de Energía y Minas del Perú: Anuarios Mineros 2000 / 2006. 